J. Lake Sci.(湖泊科学), 2018, 30(2):385-393

DOI 10. 18307/2018. 0210

(C) 2018 by Journal of Lake Sciences

\title{
水温、光能对春季太湖藻类生长的耦合影响”
}

\author{
赵巧华,孙国栋,王健健, 于志国,姜 勃 \\ (南京信息工程大学水文气象学院,南京 210044)
}

\begin{abstract}
摘 要: 环境因素对藻类生长的影响机制是探讨蓝藻水华暴发的基础, 其中水温和光能均是影响藻类生长的关键物理因 子. 基于 2015 年春季于太湖观测的 11 次藻类总初级生产力、水温廓线和营养盐浓度等,探讨水温、光能及营养盐对藻类 生长过程的影响. 结果表明: 春季, 水温、光能是影响藻类生长的关键因素, 而营养盐的影响贡献相对较弱. 深层水体中光 能是藻类生长的关键性限制因子, 浅层表现为水温、光能的共同影响, 而表层主要表现为光能的抑制. 水温的升高促进藻 类对光能的获取和利用, 提高光抑制的光能阈值, 造成深层水体中光能限制程度的加强, 藻类生长呈现光限制的深度变 浅. 本研究有利于确定气候变化下水生生态系统演变的方向, 为水生生态系统的恢复提供理论依据.
\end{abstract}

关键词: 水温;光能;藻类生长;耦合;太湖;梅梁湾;藻类总初级生产力

\section{Coupling effect of water temperature and light energy on the algal growth in Lake Taihu}

ZHAO Qiaohua, SUN Guodong, WANG Jianjian, YU Zhiguo \& JIANG Bo

( School of Hydro-Meteorology, Nanjing University of Information Science and Technology, Nanjing 210044, P.R.China)

\begin{abstract}
The effect of environmental factors on the gross primary production of phytoplankton is a basis for understanding the cyanobacteria bloom. Hereinto, water temperature and light energy are the key physical factors affecting phytoplankton growth. Based on phytoplankton primary productivity, water temperature profile and nutrient concentration observed in Lake Taihu in the spring of 2015 , we explored the effects of these environmental factors on the the algae gross primary production. Results showed that in spring, water temperature and light energy were the key factors affecting algae growth, but the effect of nutrient was relatively weak in deep layers. The light energy and water temperature jointly affected the gross primary production of phytoplankton in shallower layer. The production were inhibited by light energy in surface layer. The water temperature increase promoted the ability of algae for the acquisition and utilization of light, improved the threshold value of light limitation, and enhanced the effect of light limitation, and shallowed the photo-inhibition depth. Current study is helpful to determine the evolution direction of aquatic ecosystems under climate change, and to provide an approach for the aquatic ecosystems restoration.
\end{abstract}

Keywords: Water temperature; light energy ; algal growth; coupling; Lake Taihu; Meiliang Bay ; gross primary production of phytoplankton

内陆湖泊藻类水华频发是加剧水体环境恶化的关键因素,也是水生生态系统退化的重要表征. 藻类的 生长状态是对水体中物理、化学和生物过程的综合响应 ${ }^{[1-2]}$. 对水生生态系统的长期监测发现:太湖蓝藻优 势确立和水华首次暴发的时间存在显著的年际变化,且引起该变化的主因是风速和太阳辐射 ${ }^{[3-4]}$; 同样具备 充足营养盐水平的不同水体中,藻类的生产力也存在显著差异,其差异难以从营养盐角度得到完美的解 释 ${ }^{[3,5]}$. 相对营养盐和光能而言, 水温通过影响藻类对营养盐和光能利用进而间接影响其生长. 可见, 浅水湖 泊中水温、光能的耦合是影响蓝藻水华首次暴发的决定性因素 ${ }^{[6]}$, 即物理过程对藻类生长状态有重要影响. 因此,基于物理和化学等影响因子探讨上述差异的机制对水生生态系统治理与修复有着重要的意义.

藻类生长的物质基础是营养盐, 能量来源是光合有效辐射, 这二者直接控制藻类的生长. 水温则是另一

* 国家自然科学基金项目 (41371222,51609116)、江苏省自然科学基金项目 (BK20160961) 和中央级公益性科研院所 基本科研业务项目 (Y516034) 联合资助. 2017-05-07 收稿;2017-06-29 收修改稿. 赵巧华( 1972 ), 男, 博士, 教 授; E-mail: qhzhao@ nuist.edu.cn. 
个重要的影响因素, 水温在同化过程 (将吸收的无机营养重新合成有机物和进行能量它存的过程) 中通过影 响营养盐摄人、细胞中酶的活性等进而影响藻类生产力 ${ }^{[7]}$. 可见水温、光能及营养盐之间存在相互耦合作 用, 共同影响着藻类的初级生产力 ${ }^{[8]}$. 此耦合作用对藻类生长的影响也随水生生态系统的差异、季节变化存 在显著差异和转换 ${ }^{[9]}$ : 太湖冬、春 2 季藻类生长率受磷的限制, 而夏、秋 2 季主要受氮的限制 ${ }^{[10]}$; Dokulil 等 ${ }^{[11]}$ 认为太湖中藻类生长率主要受到光和磷的限制. 但多数探讨藻类生长对诸环境因子响应机制方面, 较 少耦合水温的效应.

就水温、光能的物理过程而言, 温度变化的时间跨度长, 其主要变化趋势体现在季节转换过程; 光能的 变化同时存在于时间和水深两个方面. 二者变化尺度的差异阻碍了通过室内模拟或野外观测探讨温、光及 其耦合对藻类生长的影响机制 ${ }^{[10-11]}$. 即使单独探讨水温和光能的耦合效应的研究也因水生生态系统的不同 而导致较大的不确定性: Duart ${ }^{[12]}$ 认为藻类生长的的最优强度独立于温度, 而 Bernard 等 $^{[13]}$ 却得到该最优光 强是温度的函数. 另一方面, 在讨论藻类光合作用的过程中, 基本忽略了水体中其他介质对光合有效辐射的 竞争, 从而可能因高估了藻类对光能的获取而导致低估藻类光合利用效率, 进而降低探讨温、光对藻类生长 影响机制的精准性. 总之, 鉴于探索温度、光能及营养盐的耦合对藻类生长状态影响存在障碍, 造成水生生 态系统中藻类生长状态对上述因子及其相互作用的普适性的定量响应机制依然不够明晰.

太湖 $\left(31^{\circ} 15^{\prime} 35.77^{\prime \prime} \mathrm{N}, 120^{\circ} 12^{\prime} 1.73^{\prime \prime} \mathrm{E}\right)$ 是典型的大型浅水富营养化湖泊, 风浪作用显著, 使得水温混合相 对均匀, 光合有效辐射的衰减作用强烈 ${ }^{[11]}$, 其水温对气象条件及太阳辐射等响应速度相对迅速, 光场垂向变 化显著, 因而春季升温期间 (4-5 月期间), 以太湖梅梁湾为研究区域, 测定藻类总初级生产力、水下光场和 水温廓线及营养盐浓度, 有利于探究水温、光能及营养盐对藻类生长的贡献及其联合影响机制, 为水生生态 系统恢复奠定理论基础.

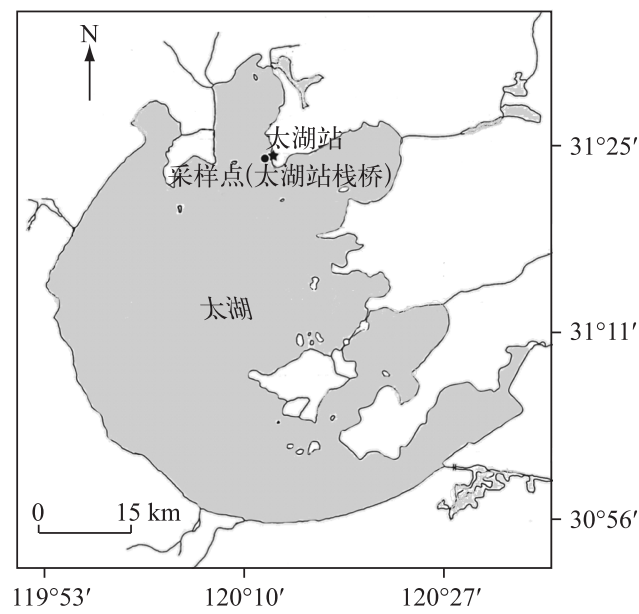

图 1 中国科学院太湖湖泊生态系统 研究站及采样点位置

Fig.1 Location of sampling site and Taihu

Laboratory of Lake Ecosystem Research, Chinese Academy of Sciences

\section{1 数据与方法}

\section{1 总初级生产力测定}

野外实验地点在中国科学院太湖湖泊生态系统观 测研究站的栈桥桥头 $\left(31.42367^{\circ} \mathrm{N}, 120.2095^{\circ} \mathrm{E}\right.$ ) (图 1). 选择晴好天气进行实验, 实验持续时段是 2015 年 4-5 月, 总计开展 11 次野外观测实验.

采用黑白瓶法测定藻类总初级生产力 ${ }^{[15-16]}$. 白瓶的 深度分别为 $0 、 10 、 20 、 30 、 40 、 50 、 60 、 80 、 100$ 和 $120 \mathrm{~cm}$, 每层 3 个平行瓶; 鉴于藻类的呼吸作用主要受水温控制 等因素 ${ }^{[14]}$, 及为了缩短挂瓶前期、收瓶固定时间以控制 实验误差, 黑瓶的深度分别设置为 $0 、 20 、 40 、 60$ 和 80 $\mathrm{cm}$, 每层 2 个平行瓶; 另设置 3 个初始瓶. 水样采集水面 下 $50 \mathrm{~cm}$ 处的水样灌到水桶中, 随后通过摚拌以保证均 匀的条件下分别罐装到溶解氧瓶中, 且保证 3 倍的溢出 体积. 初始瓶用硫酸锰和碱性碘化钾对溶解氧进行固 定. 黑白瓶曝光时间为 $10: 00-14: 00$, 曝光结束后进行 溶解氧的固定和溶解氧瓶的液封. 随后在 $30 \mathrm{~min}$ 之内到 实验室进行滴定 (滴定使用数显滴定仪, 精度为 0.01

$\mathrm{ml}$ ), 以计算白瓶、黑瓶及初始瓶的溶解氧浓度 $(\mathrm{mg} / \mathrm{L})^{[15-16]}$. 测定结果证实黑瓶中溶解氧浓度基本不随深度 变化, 因此基于各层黑瓶溶解浓度的平均值及白瓶溶解氧浓度计算藻类的总初级生产力.

\section{2 水温测定}

利用 TR 1060 水温仪测定水温,时间间隔为 $10 \mathrm{~min}$,深度间隔为 $10 \mathrm{~cm}$. 依据时间和深度计算平均水温 $T\left(T=\frac{1}{N_{n d} \cdot M_{n t}} \sum_{1}^{n t} \sum_{1}^{n d} w t(i d, i t)\right.$, 式中 $w t(i d, i t)$ 是 $i d$ 层深度和 $i t$ 时次的水温, $n t$ 为总时次数, $n d$ 为总层数 $)$. 


\section{3 水体理化指标测定}

采用碱性过硫酸钾消解紫外分光光度法、钿蓝法 (molybdenum blue method) 分别测定灌瓶水样中溶解态 总氮 ( TDN, $\mathrm{mg} / \mathrm{L}$ ) 和溶解态总磷 ( TDP, $\mathrm{mg} / \mathrm{L}$ ) 浓度 ${ }^{[17]}$, 采用 $90 \%$ 热乙醇萃取和分光光度法测定叶绿素 a 浓 度 $(\mathrm{Chl} . \mathrm{a}, \mathrm{mg} / \mathrm{L})^{[18]}$.

\section{4 水体介质吸收系数的测定}

基于 Zhang ${ }^{[19]}$ 的方法分别测定灌瓶水样中颗粒物 $\left(a_{\mathrm{p}}(\lambda)\right)$ 、非藻类颗粒物 $\left(a_{\mathrm{d}}(\lambda)\right)$ 、藻类 $\left(a_{\mathrm{ph}}(\lambda)\right)$ 和有 色溶解有机质 $\left(a_{\mathrm{g}}(\lambda)\right)$ 的吸收系数. 纯水的吸收系数 $\left(a_{\mathrm{w}}(\lambda)\right)$ 来自 Smith and Baker ${ }^{[20]}$ 测定的数据, 由此依据 吸收系数的可加性, 计算出水体的总吸收系数 $\left(a_{\mathrm{t}}(\lambda)\right)$ 和藻类吸收系数与总吸收系数的比例 $(\alpha(\lambda))$. 各吸 收系数均在光合有效辐射范畴 ( $400 \sim 700 \mathrm{~nm})$.

\section{5 水下标量辐照度廓线的计算}

$$
\begin{gathered}
a_{\mathrm{t}}(\lambda)=a_{\mathrm{p}}(\lambda)+a_{\mathrm{g}}(\lambda)+a_{\mathrm{w}}(\lambda) \\
\alpha(\lambda)=a_{\mathrm{ph}}(\lambda) / a_{\mathrm{t}}(\lambda)
\end{gathered}
$$

用 TRIOS 水下光谱仪测定水体各层的上、下行辐照度 $E_{\mathrm{d}}(\lambda, z)$ 和 $E_{\mathrm{u}}(\lambda, z)$, 测定时间间隔为 $30 \mathrm{~min}$, 深度 间隔为 $10 \mathrm{~cm}$. 鉴于真实表征水下光能转换的物理量 (包括转换为生物能和热能) 为标量辐照度 $\left(E_{\mathrm{o}}(\lambda, z)\right)^{[21]}$, 为此需计算标量照度的廓线分布, 以进一步通过波长的积分计算各曝光时段各深度的藻类所吸收光合有效 辐射的光量子数 $(\phi(z, t))$. 依据 Gershun 定律 ${ }^{[22]}$ 计算各深度的标量辐照度, 并随之计算 $\phi(z, t)$.

$$
\begin{aligned}
E_{\mathrm{o}}(\lambda, z) & =-\frac{1}{a_{\mathrm{t}}(\lambda)} \frac{\mathrm{d}\left[E_{\mathrm{d}}(\lambda, z)-E_{\mathrm{u}}(\lambda, z)\right]}{\mathrm{d} z} \\
\phi(z, t) & =\int_{\lambda=400}^{\lambda=700} E_{\mathrm{o}}(\lambda, z) \cdot a_{\mathrm{ph}}(\lambda) \cdot \mathrm{d} \lambda
\end{aligned}
$$

在每日黑白瓶曝光时段内进行 9 次水下光场的测定,因而需将 $\phi(z, t)$ 在 10:00-14:00 时段内进行插 值, 随之对时间进行积分得出各层次曝光时段藻类吸收的光量子数 $\phi(z)$.

\section{2 结果}

\section{1 藻类总初级生产力 $(P)$ 与光能 $(I)-$ 水温 $(T)$ 的拟合}

依据总初级生产力-光能-水温 $(\mathrm{P}-\mathrm{I}-\mathrm{T})$ 相互作用的基本原理, 采用 Duarte ${ }^{[12]}$ 的模拟公式对 11 次的总 初级生产力与光廓线进行非线性拟合. 鉴于每次测定总初级生产力时藻类的生物量有差异, 因而为探究水 体总初级生产力廓线的变化机制, 将总初级生产力和藻类吸收光量子数及各营养盐浓度分别除以叶绿素 a 浓度, 即表征单位叶绿素的藻类吸收的光量子数和水体中营养盐浓度, 均归一化到单位叶绿素 a 的藻类. 为 简便起见, 以下提到的藻类吸收的光量子数、藻类的总初级生长力、藻类的最大总初级生产力、各营养盐浓 度均以单位叶绿素 $\mathrm{a}$ 的藻类为标准.

$$
\begin{gathered}
P_{\mathrm{NZ}}\left(\phi_{\mathrm{n}}(z), T\right)=a_{0}+a_{1} \cdot \phi_{\mathrm{n}}(z)+a_{2} \cdot T+a_{3}\left[\phi_{\mathrm{n}}(z)\right]^{2}+a_{4} \cdot T^{2}+a_{5} \cdot \phi_{\mathrm{n}}(z) \cdot T \\
\phi_{\mathrm{n}-\text { opt }}=-\frac{a_{1}+a_{5} \cdot T}{2 a_{3}} \\
P_{\mathrm{NZ}}\left[\phi_{\mathrm{n}}(z)\right]=P_{\mathrm{NMAX}} \frac{\phi_{\mathrm{n}}(z)}{\phi_{\text {nopt }}} \exp \left[1-\frac{\phi_{\mathrm{n}}(z)}{\phi_{\mathrm{nopt}}}\right]
\end{gathered}
$$

式中, $\phi_{\mathrm{n}}(z)$ 是深度为 $z$ 处藻类吸收的光量子数 $(\mathrm{mol}), P_{\mathrm{NZ}}$ 为总初级生产力 (以溶解氧释放量为单位, $((\mathrm{mg} / \mathrm{L}) /(\mu \mathrm{g} /(\mathrm{L} \cdot 4 \mathrm{~h}))) \cdot \phi_{\mathrm{n}-\mathrm{opt}}$ 为单位藻类的最优光量子数; $P_{\mathrm{NMAX}}$ 为每次最大总初级生产力, 即在实验 的水温、营养盐浓度及藻类获取最优光量子数条件下的最大总初级生产力 $((\mathrm{mg} / \mathrm{L}) /(\mu \mathrm{g} /(\mathrm{L} \cdot 4 \mathrm{~h}))) ; a_{0}$ 、 $a_{1} 、 a_{2} 、 a_{3} 、 a_{4} 、 a_{5}$ 为拟合常数.

鉴于太湖是一大型浅水湖泊, 风浪作用显著, 混合作用强烈, 水温在垂向上分布相对均匀, 且实验过程 中藻类总初级生产力是整个实验时段水温、营养盐及在各深度处所获光能的综合体现, 因而每次实验过程 的拟合, 主要体现了藻类吸收的光量子数及藻类的产氧量存在的垂向变化, 没有体现小时尺度上的时间变 化. 可见,尽管单次拟合表面上类似于 Eiler 的拟合 ${ }^{[23]}$,但是,拟合公式表征了各实验过程水温、营养盐、各深 
度处所获光量子数的综合作用, 通过多次拟合结果之间的比较可以反映水温、光能对藻类生长的影响规律.

图 2 展示了春季 11 次观测结果的拟合, 其拟合效果均较好 (表 1). 图 2 表明:在低光区藻类总初级生产 力随吸收的光量子数增加呈线性增加, 随后斜率减小逐渐达到饱和状态, 除少数观测外 (4 月 16 日), 绝大部 分随着光量子数的增大呈现出光抑制现象. 就光抑制而言, 其出现深度基本处于 $10 \mathrm{~cm}$ 以上的水体中表层; 光饱和现象主要呈现在 $10 \sim 20 \mathrm{~cm}$ 的次表层. 另外, 藻类光饱和时的藻类吸收的光量子数和光抑制程度不尽 相同,说明藻类总初级生产力的光抑制现象不仅仅受光能的影响,同时还受到其他因素的影响.
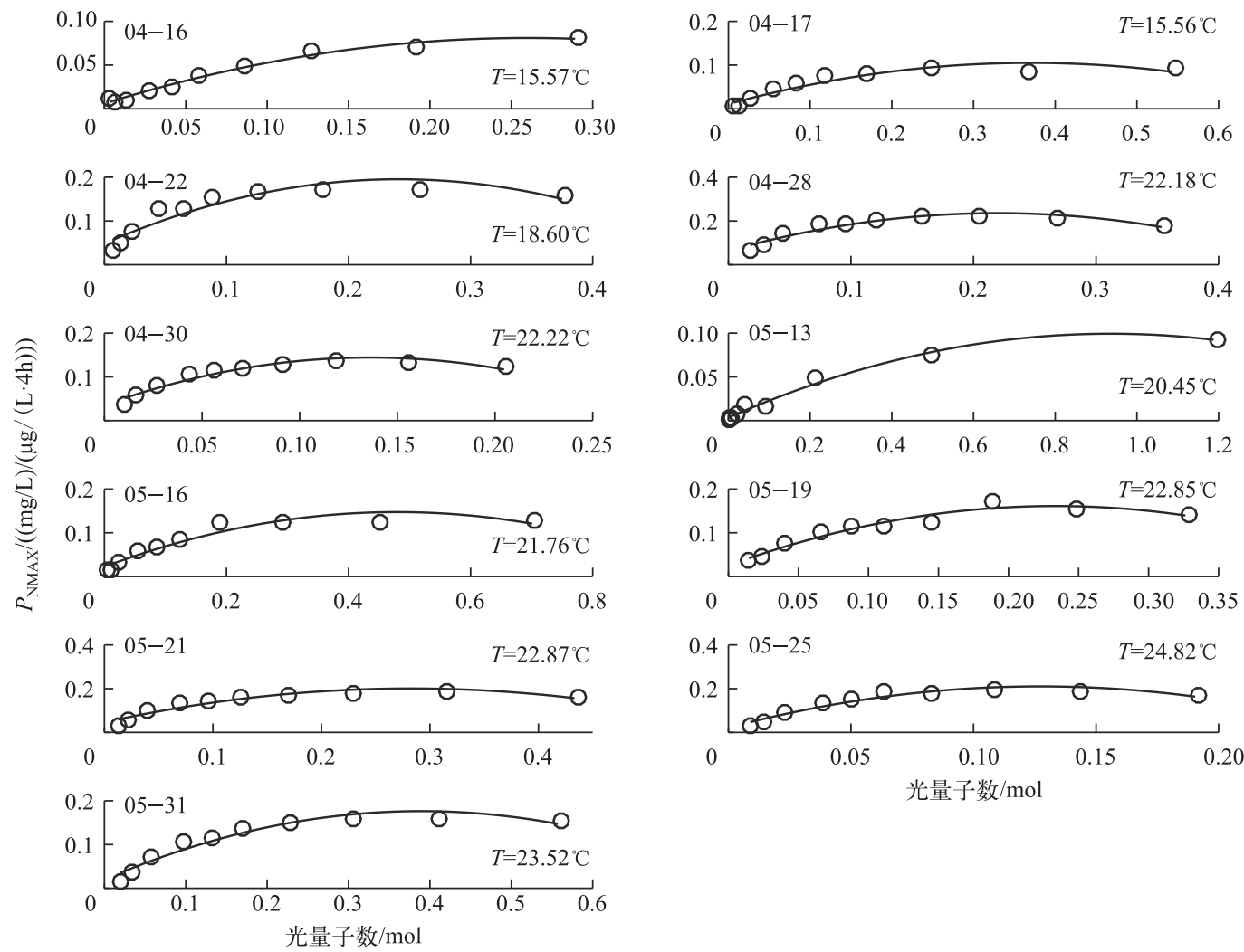

图 2 藻类总初级生产力与水温、光能关系的拟合

Fig.2 Fitting for relationships among the gross primary production and water temperature and light energy

表 1 野外实验拟合效果 *

Tab.1 Fitting effect for the experiments

\begin{tabular}{|c|c|c|c|c|c|c|c|c|c|c|c|}
\hline & \multicolumn{11}{|c|}{ 日期／(月－日 ) } \\
\hline & $04-16$ & $04-17$ & $04-22$ & $04-28$ & $04-30$ & $05-13$ & $05-16$ & $05-19$ & $05-21$ & $05-25$ & $05-31$ \\
\hline 样本数 & 10 & 10 & 10 & 10 & 10 & 10 & 10 & 10 & 10 & 10 & 10 \\
\hline 相关系数 & 0.992 & 0.944 & 0.937 & 0.955 & 0.968 & 0.993 & 0.965 & 0.971 & 0.952 & 0.973 & 0.970 \\
\hline$P_{\text {NMAX }}$ & 0.0810 & 0.1051 & 0.1954 & 0.2357 & 0.1443 & 0.0993 & 0.1473 & 0.1611 & 0.2001 & 0.210 & 0.1764 \\
\hline 置信度 & 0.99 & 0.99 & 0.99 & 0.99 & 0.99 & 0.99 & 0.99 & 0.99 & 0.99 & 0.99 & 0.99 \\
\hline
\end{tabular}

$* P_{\text {NMAX }}$ 的单位为 $(\mathrm{mg} / \mathrm{L}) /(\mu \mathrm{g} /(\mathrm{L} \cdot 4 \mathrm{~h}))$.

\section{2 影响藻类总初级生产力的关键因素分析}

除了光因素外, 水温、营养盐等也是影响藻类总初级生产力的关键因子. 可见, $P_{\text {NMAX }}$ 是实验水温、营养盐 
条件下藻类获取最优光量子数时的最大总初级生产力, 是对实验时段的水温、营养盐的综合响应, 因此用总 初级生产力作为因变量, 水温、溶解态总氮、溶解态总磷作为自变量进行逐步回归, 以进一步探究影响春季 太湖藻类生长的关键因素. 逐步回归的线性方程为:

$$
P_{\mathrm{NMAX}}=-0.072+0.011 T
$$

该拟合是通过 SPASS 13.0 软件完成, 置信性水平为 $0.02(F=7.680)$, 拟合结果证实了太湖春季藻类生 长除光能外主要受水温的控制, 而营养盐对其影响相对较弱.

\section{3 温度、光能对藻类总初级生产力的影响机制}

鉴于实验室模拟实验难以同时模拟与野外相同光质的光合有效辐射、整个季节的水温变化对藻类生长 的影响,但本文所利用的拟合公式呈现了水温、光能的联合效应,因此可基于拟合公式探讨水温、光能对藻 类总初级生产力影响的耦合效应.
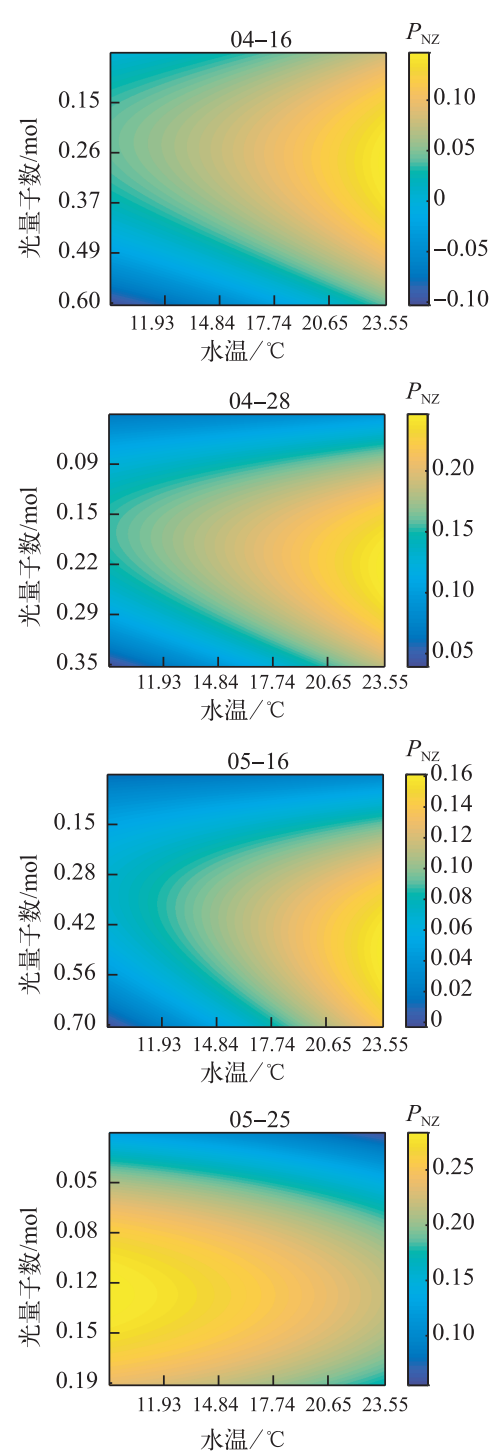
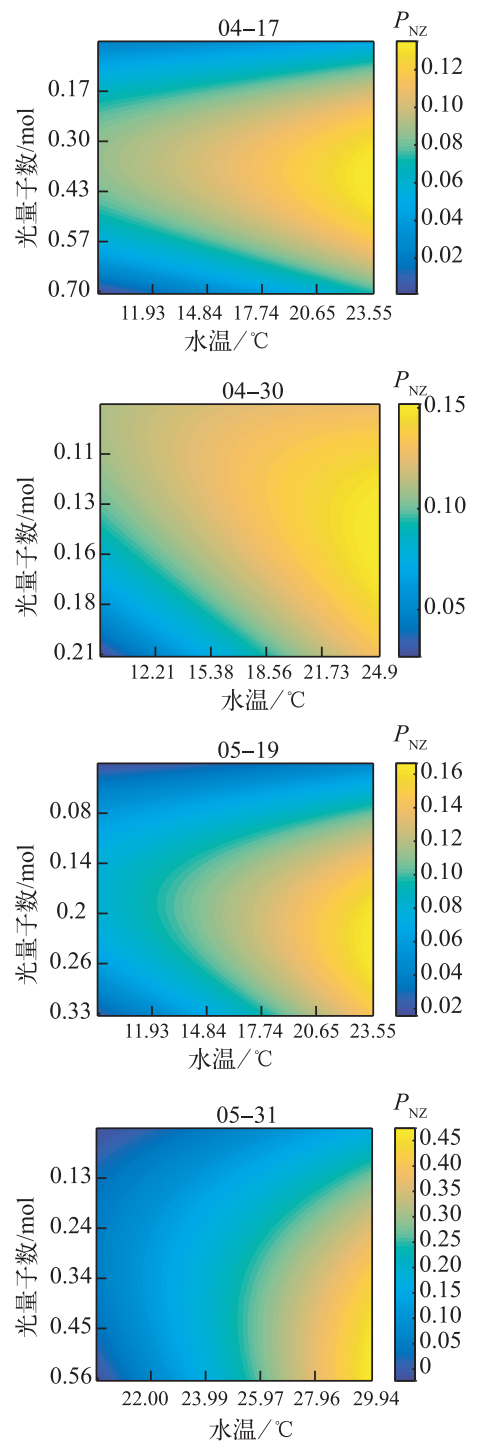
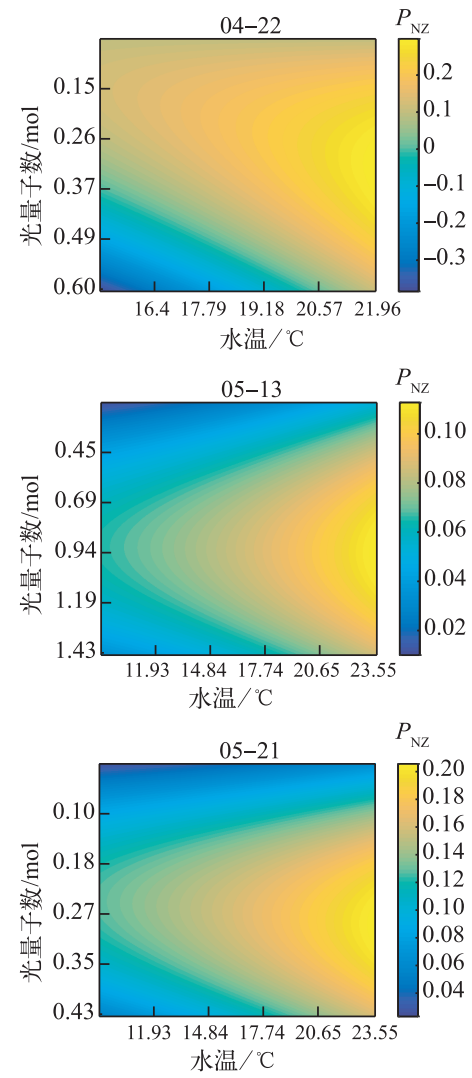

图 3 水温、光能对藻类总初级生产力的影响

Fig. 3 The effect of water temperature and light energy on the gross primary production of phytoplankton 
依据野外实验得到的 11 个拟合公式, 对不同水温及藻类获取的光量子数进行模拟 (图 3). $P_{\mathrm{NZ}}$ 随单位叶 绿素获取的光量子数的增大呈现先增大后减小的变化趋势, 呈单峰型, 既展现了在光限制状态下光合有效 辐射对藻类生长的促进作用, 也呈现了随光合有效辐射的进一步增大造成的光抑制现象. 除 5 月 25 日外,

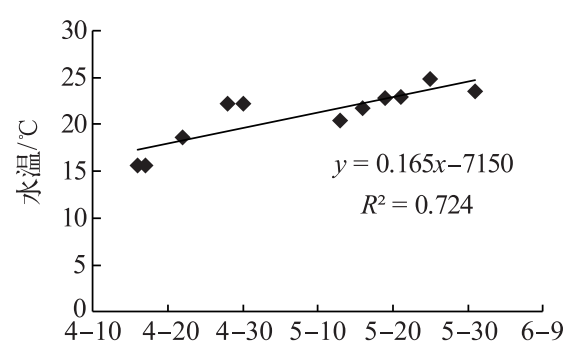

图 4 水温随时间的变化

Fig. 4 The variation of water temperature with time 藻类总初级生产力随水温升高而增大,而在 5 月 25 日的实验 呈现出水温对藻类生长的抑制作用.

本文在 2.2 节中探讨了影响藻类总初级生产力的关键控 制因子是光能和水温,但该结果是基于 $4-5$ 月 11 次实验的 总体趋势特征. 然而就某一次具体的实验而言,依然需要从其 营养盐浓度及水温等角度进行分析: 在整个实验时段内, 5 月 25 日的水温最高 (图 4), 因而从水温的角度而言, 藻类的生长 需要更多的营养盐和光能支持, 但由于 TDN 浓度最小、TDP 浓度排倒数第二 (表 2), 造成营养盐不足以支持藻类生长的 需要,因而可能造成即使在低光能供给的条件下,水温往往呈 现对藻类生长的抑制.

表 2 水体中营养盐浓度

Tab. 2 Concentrations of nutrients in the water

\begin{tabular}{lccccccccccc}
\hline \multirow{2}{*}{ 营养盐 } & $\begin{array}{c}4 \text { 月 } \\
16 \text { 日 }\end{array}$ & $\begin{array}{c}4 \text { 月 } \\
17 \text { 日 }\end{array}$ & $\begin{array}{c}4 \text { 月 } \\
22 \text { 日 }\end{array}$ & $\begin{array}{c}4 \text { 月 } \\
28 \text { 日 }\end{array}$ & $\begin{array}{c}\text { 4月 } \\
\text { 30 }\end{array}$ & $\begin{array}{c}\text { 5月 } \\
13 \text { 日 }\end{array}$ & $\begin{array}{c}\text { 5月 } \\
16 \text { 日 }\end{array}$ & $\begin{array}{c}\text { 5月 } \\
19 \text { 日 }\end{array}$ & $\begin{array}{c}\text { 5月 } \\
21 \text { 日 }\end{array}$ & $\begin{array}{c}\text { 5月 } \\
25 \text { 日 }\end{array}$ & $\begin{array}{c}5 \text { 月 } \\
31 \text { 日 }\end{array}$ \\
\hline $\mathrm{TDN} /(\mathrm{mg} / \mathrm{L})$ & 0.2138 & 0.2242 & 0.1596 & 0.3544 & 0.1307 & 0.1316 & 0.1998 & 0.1501 & 0.1511 & 0.1139 & 0.2448 \\
$\mathrm{TDP} /(\mu \mathrm{g} / \mathrm{L})$ & 2.2327 & 1.3697 & 0.7281 & 2.8363 & 1.2264 & 1.8392 & 3.4551 & 2.2043 & 2.6492 & 1.3122 & 4.1465 \\
\hline
\end{tabular}

藻类的最大总初级生产力随水温升高向高光区过渡, 可见水温有促进藻类对光的利用能力, 提高藻类 出现光抑制现象的阈值, 弱化光对藻类生长的抑制作用, 这表明春季太湖水温对藻类光合作用的影响主要 通过影响藻类对光的利用而表现出来. 此外, 藻类最大总初级生产力随水温逐渐升高, 但各自升高的程度不 尽相同. 另一方面光能对水温的影响机制较为复杂, 主要表现在水一气之间的感热、潜热、长波辐射及太阳短 波辐射的效应 ${ }^{[24]}$, 本文对此不作深人讨论.

\section{4 藻类总初级生产力对水温、光能的敏感性}

水温和光能均是影响藻类的关键因素, 且存在相互影响, 厘清二者的贡献及其变化规律对于理解蓝藻 水华暴发机制有着重要的作用. 本文参照 Cloern 的方法 ${ }^{[25]}$, 基于 $P_{\mathrm{NAMX}}$ 对 $\phi_{n}(z)$ 和 $T$ 的偏导数讨论光能、水 温对藻类总初级生产力的影响规律. 二者之间的比值 $R$ (无量纲量) 在 $-0.1 \sim 0.1$ 区间, 表征藻类总初级生产 力主要受温度影响控制; $R$ 介于 $0.1 \sim 10$ 或 $-10 \sim-0.1$ 区间, 受水温、光能的共同影响; 而 $R>10$ 或 $R<-10$, 表 征藻类获取的光量子数对其总初级生产力的影响程度远大于温度的效应: 当水温升高促进藻类生长时, $R>$ 10 表征光限制, 而 $R<-10$ 表征光抑制; 当水温升高抑制藻类生长时, $R$ 表征的光限制与抑制亦相反.

依据拟合公式建立的藻类总初级生产力对水温、光能的响应规律可以分为 2 类 (图 5). 其一, 本文的大 部分水温、光能实验, 光能对藻类的限制作用随温度升高逐步增大, 而光的抑制作用逐步减弱; 在水温、光能 对藻类生长的影响区域中,随着水温升高, 水温、光能共同影响的区域相应增大; 其二, 5 月 13 日和 5 月 25 日, 藻类总初级生产力对水温、光能响应的趋势随水温没有明显的变化趋势, 5 月 25 日, 水温对藻类生长呈 现抑制作用, 因而其光限制、抑制作用呈现相反的比值; 无论哪种类型, 水温、光能对藻类总初级生产力的影 响程度也不尽相同. 这也证明了春季水温逐步提高, 在一定范围内温度会加剧光对藻类生长的限制.

\section{3 讨论}

本文基于春季水下光谱、藻类总初级生产力及水温廓线、营养盐的观测, 探讨水温、光能对春季太湖藻 类生长的耦合影响. 实验采用封闭的环境可能会造成溶氧瓶中营养盐短缺, 且未考虑水一土界面的营养盐交 

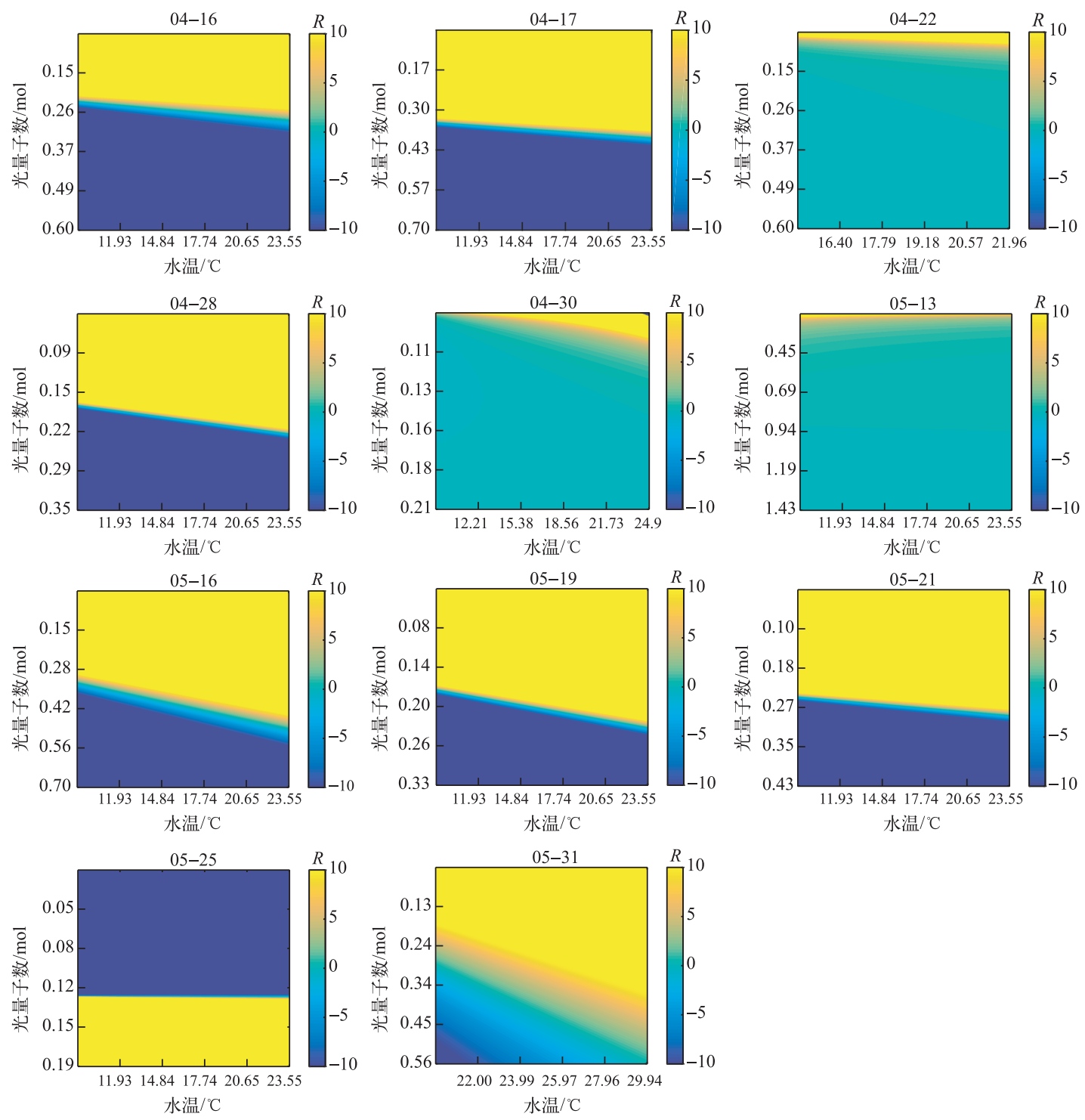

图 5 藻类总初级生产力对水温、光能的敏感性

Fig.5 The sensitiveness of the gross primary production of phytoplankton to water temperature and light energy

换对藻类总初级生产力产生影响, 同时, 为了尽量减小上述影响, 本文采用短曝光时间的方式 ${ }^{[10]}$. 另一方 面, 由于水温较低且光能不足的情况下藻类总初级生产力较弱, 因此, 误差可能会增大, 而导致观测时间均 选择天气晴好且光线最强的 $10: 00-14: 00$ 时段,力求将误差控制到最小的范围.

藻类的总初级生产力是物理、化学及生物过程的综合反应. 其中诸因子不仅存在时间变化, 同时也存在 空间变化. 就单次实验而言,空间变化主要体现在藻类获取的光量子数和初级生产力在垂向上的变化,而水 温及营养盐在空间上均匀,这也在一定程度上说明基于 P-T-I 曲线中获取的单位叶绿素的最大总初级生产 力涵盖了实验过程中营养盐和温度的信息. 本文的系列实验数据不仅包含了光能差异, 同时也涵盖水温和 营养盐的变化 (4-5 月), 因而逐步回归能够证实除光能外水温是影响藻类总初级生产力的关键因子. Xu 等 ${ }^{[10]}$ 和 $\mathrm{Ma}$ 等 ${ }^{[26]}$ 讨论了不同季节营养盐及其相互作用对藻类生长率的影响, 但该结果不能体现藻类总初级 生产力对水温的响应, 难以准确描述春季由于水温升高对藻类的影响效应, 而本文一系列实验能体现出各 
环境要素的综合影响, 所得的结论相对较为全面. 此外, 由于每次实验藻类的生物量有差异, 判断水体中营 养盐浓度是否限藻类生长应量化到单位叶绿素. 因此基于单位叶绿素获取的光量子数、溶解态总氮 (磷) 和 水温的分析来探讨影响藻类生长的关键因素更为科学和可靠.

水温是通过影响藻类酶的活性间接对光能和营养盐的获取、利用产生影响, 进而影响藻类的生长 ${ }^{[7]}$. 本 研究的实验时段 ( 4 月上旬至 5 月底), 太湖春季水温最低为 $15.6^{\circ} \mathrm{C}$ ( 4 月 16 日), 最高水温为 $24.8^{\circ} \mathrm{C}(5$ 月 25 日), 水温随时间基本呈上升趋势 (图 4). 各种群藻类生长的最适宜温度基本均大于 $25^{\circ} \mathrm{C}^{[27]}$, 结合春季水温 的变化范围可知, 春季水温对藻类生长的影响呈限制状态, 即春季水温作用通过限制藻类对营养盐摄人、光 合有效辐射能量获取及同化作用而限制藻类的总初级生产力, 从而也降低藻类对营养盐的需求, 弱化了营 养盐对藻类生长的反馈作用, 加之太湖属于重度富营养化湖泊 ${ }^{[10]}$ 、水温限制藻类生长的状态下, 营养盐浓度 完全能满足春季藻类的生长需求, 使得水温和光能成为影响春季太湖藻类生长的关键过程, 而营养盐的贡 献相对较弱. 此结果与 Chen 等 $^{[6]}$ 和 Bernard 等 $^{[13]}$ 阐述的无营养盐限制条件下,水温、光能对藻类生长和蓝 藻水华首次暴发起关键作用的结论相吻合.

水温、光能对春季太湖藻类生长既有促进作用, 也有抑制作用 ${ }^{[13]}$, 且两者也存在耦合的作用机制. 鉴于 光合有效辐射随传输深度呈现 $\mathrm{e}$ 负指数递减, 藻类在与其他光学介质竞争中获取光量子数也呈 $\mathrm{e}$ 指数递减. 在整个野外观测实验中, 大部分藻类生长力在 $0.2 \sim 0.3 \mathrm{~m}$ 处出现光饱和状态, 而在表层发生抑制现象; 随深 度的增加, 藻类生产力受到光能限制, 而且随深度的递增, 藻类生产力与其获取的光量子数的关系为线性关 系 (图 2), 即光是限制藻类生长的关键因素 ${ }^{[28]}$. 总之, 在整个春季水温上升的过程, 呈现出对藻类生长的促 进作用, 而对藻类生产力深度廓线而言, 存在明显的光限制现象, 因而水温和光能对藻类生长的影响分别体 现在时间和空间上.

鉴于藻类获取的光量子数随深度快速递减 (图 2), 造成实验时段内营养盐和温度条件下弱光区的藻类 生长受到光限制, 随深度变浅藻类获取的光量子数增加, 光能造成的限制作用逐渐变弱, 从而表现出藻类生 长的光饱和及抑制作用. 春季的 11 次实验过程中伴随着温度的升高, 藻类酶的活性增加, 藻类生长能力得 以提高, 其对光能的需求增加, 造成达到光饱和的阈值提高, 也进一步增强了光限制的程度 (图 5 , 除 5 月 25 日), 而 5 月 25 日光能的负反馈作用可能与其营养盐浓度最低有关.

水温、光能对藻类生长的影响随深度而变化. 随着水深的增加, 水体中光能减弱, 使得藻类获取的光量 子数也随深度减小. 藻类生长缺乏能量来源, 表现出对光能的强烈依赖性 (或敏感性) 增强、藻类总初级生产 力与光能呈线性关系 ${ }^{[28]}$ (图 2、5); 另一方面, 随着水深变浅, 藻类获取的光量子数增大, 对光能的依赖作用 减弱, 逐步表现出对水温和光能的共同依赖, 随着藻类获取的光能进一步增大, 藻类的生长过程中, 由于春 季温度未达到最优温度, 即藻类生长的潜力也因温度限制难以达到对应营养盐浓度下的最大值, 因而其在 生长过程难以完全利用所吸收的光量子, 甚至造成藻类的光合作用呈现光饱和甚至抑制现象, Zhou ${ }^{\text {等 }}{ }^{[29]}$ 也 发现光抑制现象在太湖中也是常见现象.

\section{4 结论}

阐明影响湖泊中藻类生长的关键因素, 对水生生态系统的恢复有着重要的意义. 本文基于春季 11 次野 外观测的藻类初级生产力、水温廓线、水下光谱及营养盐浓度等数据, 探讨了影响藻类生产力的关键因素, 及水温、光能对太湖春季藻类总初级生产力的耦合影响. 主要结论如下:

1) 春季太湖藻类生长主要受水温和光能的控制, 营养盐的贡献相对较弱.

2) 深层水体中光能是限制藻类生长的关键因素, 浅层水体中水温和光能共同限制藻类的生长, 表层水 体的光能可能抑制藻类的生长.

3) 太湖水体对光能传输的强衰减作用造成藻类生长受光的限制,且主要体现在空间的垂向上,而水温 的升高促进光能对藻类生长的限制作用加强、抑制作用减弱,造成藻类光抑制的阈值升高.

\section{5 参考文献}

[ 1 ] Paerl HW, Fulton RSIII, Moisander PH et al. Harmful freshwater algal blooms, with an emphasis on cyanobacteria. The 
Scientific World J, 2001, (1) : 76-113.

[ 2 ] Song LR, Zhang T, Zheng LL. Why cyanobacteria blooms raging? Life World, 2007, 34(8) : 36-41.

[ 3 ] Zhang M, Duan HT, Shi XL et al. Contributions of meteorology to the phenology of cyanobacterial blooms: Implications for future climate change. Water Research, 2012, 46: 442-452.

[ 4 ] Duan HT, Ma RH, Xu XF et al. Two-decade reconstruction of algal blooms in China's Lake Taihu. Environment Science and Technology, 2009, 43: 3522-3528.

[ 5 ] Yamaguchi H, Hirade N, Higashizono $\mathrm{K}$ et al. Light and nutrient limition on phytoplankton production in the strait of an enclosed coastal sea(Bisan Strait, eastern Seto Inland Sea, Japan). Journal of Sea Research, 2015, 103: 75-83.

[ 6 ] Chen M, Fan M, Liu R et al. The dynamics of temperature and light on the growth of phytoplankton. Journal of Theoretical Biology, 2015, 385: 8-19.

[ 7 ] Thorel M, Fauchot J, Morelle $\mathbf{J}$ et al. Interactive effect of irradiance and temperature on growth and domoic acid production of the toxic diatom Pseudo-nitzschia australis (Bacillariophyceae). Harmful Algae, 2014, 39: 232-241.

[ 8 ] Zhou Y, Geng L, Wang W et al. Combined effect of temperature, light intensity, and nitrogen concentration on the growth and polysaccharide content of Microcystis aeruginosa in batch culture. Biochemical Systematics and Ecology, 2012, 41: 130-135.

[ 9 ] Dubourg P, North R, Hunter K. Light and nutrient co-limitaton of phytoplankton communities in a large reservoir: Lake Diefenbaker, Saskatchewan, Canada. Journal of Great Lakes Research, 2015, Supplement: 1-15.

[10] Xu H, Paerl HW, Qin B et al. Nitrogen and phosphorus inputs control phytoplankton growth in eutrophic Lake Taihu, China. Limnol Oceanogr, 2010, 55(1): 420-432.

[11] Dokulil MT, Teubner K. Cyanobacterial dominance in lakes. Hydrobiologia, 2000, 438(1/2/3): 1-12.

[12] Duarte P. A mechanism model of the effects of light and temperature on algal primary productivity. Ecological Modelling, $1995,82: 151-160$.

[13] Bernard O, Rémond B. Validation of a simple model accounting for light and temperature effect on microalgal growth. Bioresource Technology, 2012, 123: 520-527.

[14] Herb WR, Stefan HG. Integral growth of submersed macrophytes in varying light regimes. Ecological Modelling, 2003, 168: $77-100$.

[15] Aristegui J, Monero MF, Ballesteros S et al. Planktonic primary production and microbialrespiration measured by 14C assimilation and dissolved oxygen changes in coastal waters of the Antarctic Peninsula during austral summer: Implications for carbon flux studies. Marine Ecology Progress Series, 1996, 132 : 191-201.

[16] Strickland JDH. Measuring the production of marine phytoplankton. Bull Flsh Res Bd Can, 1960, 122: 122-129.

[17] APHA American Public Health Association, Standard methods for the examination of water and wastewater. 19th ed. APHA, American Water Works Association, Water Environment Federatio, 1995.

[18] Papista E, Acs E, Boeddi B. Chlorophyll-a determination with ethanol-A critical test. Hydrobiologia, 2002, 485: 191-198.

[19] Zhang YL, Qin BQ, Zhu GW et al. Effect of sediment resuspension on underwater light field in shallow lakes in the middle and lower reaches of the Yangtze River: A case study in Longgan Lake and Taihu Lake. Science in China Series D-Earth Sciences, 2006, 49(Supp I) : 114-125.

[20] Smith RC, Baker KS. Optical properties of the clearest natural waters (200-800 nm). Applied Optics, 1981, 20: 177-184.

[21] Kirk John TO. Light \& photosynthesis in aquatic ecosystems. London: Cambridge University Press, 1994: 1-528.

[22] Voss KJ. Use of the radiance distribution to measure the optical absorption coefficient in the ocean. Limnol Oceanogr, $1989,34(8):$ 1614-1622.

[23] Eiler PHC, Peeters JCH. A model for the relationship between light intensity and the rate of photosynthesis in phytoplankton. Ecological Modelling, 1988, 42: 199-215.

[24] Zhao QH, Sun JH. The variation features of the surface mixed layer depth in Erhai Lake and Taihu Lake in spring and autumn and their mechanism analyses. Acta Phys Sin, 2013, 62(3) : 039203.

[25] Cloern JE. The relative importance of light and nutrient limitation of phytoplankton growth: A simple index of coastal ecosystem sensitivity to nutrient enrichment. Aquat Ecol, 1999, 33: 3-16.

[26] Ma J, Qin B, Pan W et al. Controlling cyanobacterial blooms by managing nutrient ratio and limitation in a large hypereutrophic lake: Lake Taihu, China. Journal of Environmental Sciences, 2015, 27(1) : 80-86.

[27] Butterwick C, Heaney SI, Talling JF. Diversity in the influence of temperature on the growth rates of freshwater alage, and its ecological relevance. Freshwater Biology, 2005, 50: 291-300.

[28] Béchet Q, Shilton A, Guieysse B. Modeling the effect of light and temperature on algae growth: State of the art and critical assessment for productivity prediction during outdoor cultivation. Biotechnology Advance, 2013, 31 : 1648-1663.

[29] Zhou Q, Chen W, Shan K et al. Influence of sunlight on the proliferation of cyanobacterial blooms and its potential applications in Lake Taihu, China. Journal of Environmental Science, 2014, 26: 626-635. 